\title{
Tolerability of trivalent inactivated influenza vaccine among pregnant women, 2015
}

\author{
Suvanna Asavapiriyanont ${ }^{1}$, Wanitchaya Kittikraisak ${ }^{2 *}$, Piyarat Suntarattiwong ${ }^{3}$, Darunee Ditsungnoen², \\ Surasak Kaoiean ${ }^{1}$, Podjanee Phadungkiatwatana', Nattinee Srisantiroj ${ }^{1}$, Tawee Chotpitayasunondh³, \\ Fatimah S. Dawood ${ }^{4}$ and Kim A. Lindblade ${ }^{2,4}$
}

\begin{abstract}
Background: Thailand recommends influenza vaccination among pregnant women. We conducted a cohort study to determine if the prevalence of adverse events following immunization (AEFIs) with influenza vaccine among Thai pregnant women was similar to that often cited among healthy adults.

Methods: Women who were $\geq 17$ gestational weeks and $\geq 18$ years of age were recruited. Demographic and health history data were collected using structured questionnaires. Women were provided with symptom diary, ruler to measure local reaction(s), and thermometer to measure body temperature. AEFIs were defined as any new symptom/abnormality occurring within four weeks after vaccination. The diaries were abstracted for frequency, duration, and level of discomfort/inconvenience of the AEFIs. Serious adverse events (SAEs) and the likelihood of AEFIs being associated with vaccination were determined using standard definitions.
\end{abstract}

Results: Among 305 women enrolled between July-November 2015, median age was 29 years. Of these, 223 (73\%) were in their third trimester, 271 (89\%) had completed secondary school or higher, and 20 (7\%) reported $\geq 1$ preexisting conditions. AEFIs were reported in 134 women (44\%; 95\% confidence interval [CI] 38-50\%). Soreness at the injection site (74, 24\%; Cl 19-29\%), general weakness (50, 16\%; Cl 12-21\%), muscle ache (49, 16\%; Cl 12-21\%), and headache (45, 15\%; Cl 1-19\%) were most common. Of those with AEFIs, 120 (89\%) reported symptom/abnormality occurred on day 0 or day 1 following vaccination. Ten women (7\%) reported the AEFIs affected daily activities. The AEFIs generally spontaneously resolved within $24 \mathrm{~h}$ of onset. There were two vaccine-unrelated SAEs. Of 294 women with complete follow-up, 279 (95\%) had term deliveries, 12 (4\%) had preterm deliveries, and 3 (1\%) had miscarriage or stillbirth.

Conclusion: In our cohort, AEFIs with influenza vaccine occurred with similar frequency to those reported among healthy adults in other studies, and were generally mild and self-limited. No influenza vaccine-associated SAEs were identified.

Keywords: Tolerability, Influenza, Vaccination, Pregnant women, Thailand

\section{Background}

Pregnant women are at high risk of hospitalization from influenza virus infection, with the highest rates of influenza-associated hospitalization documented in the third trimester [1-5]. Influenza vaccination during pregnancy is safe for both the mother and fetus [6-10], and has been shown to be effective at preventing influenza in

\footnotetext{
* Correspondence: glr9@cdc.gov

${ }^{2}$ Influenza Program, Thailand Ministry of Public Health - U.S. Centers for

Disease Control and Prevention Collaboration, Nonthaburi, Thailand

Full list of author information is available at the end of the article
}

pregnant women and among their infants in the first few months of life [11-15]. The World Health Organization (WHO) recommends pregnant women receive seasonal influenza vaccine and suggests that countries looking to initiate or expand their influenza vaccination program prioritize pregnant women [1].

In 2009, the Thailand Ministry of Public Health (MOPH) issued a recommendation that pregnant women should receive influenza vaccine after the third month of pregnancy [2]. However, influenza vaccination coverage among 
pregnant women remains suboptimal in Thailand. A recent analysis by Owusu et al. revealed very low uptake of influenza vaccine in Thai pregnant women: $1.1 \%$ in 2010 , $0.7 \%$ in 2011 , and $0.9 \%$ in 2012 [16]. These data indicate that the national policy on influenza vaccination in pregnant women is not widely implemented. While a limited supply of free vaccine may be a contributor to the low coverage rates, the same analysis found significantly higher vaccination rates in persons with chronic disease $(9-14 \%)$ and those aged 65 years and older (11-20\%). The lower vaccination rates in pregnant women could be due to operational issues, but concerns by both pregnant women and their clinicians about the safety of the influenza vaccine during pregnancy may also limit vaccination rates [6].

While data from multiple studies have shown the influenza vaccine to be safe and well-tolerated during pregnancy and for the children aged $\geq 6$ months [7-11, $17,18]$, similar data are not available from Thailand. Adverse event following immunization (AEFI) reporting has been included as part of Thailand's surveillance and investigation system since 2003 in order to improve the safety and performance of the national vaccination program which covers all vaccinations provided in the country [19]. However, the database includes very few pregnant women who received influenza vaccination and relies solely on passive reporting. As a result, the local evidence base for the safety of the influenza vaccine among pregnant women in Thailand remains limited. We conducted a prospective cohort study of Thai women who received influenza vaccination during the second or third trimesters of pregnancy to systematically document the frequency of AEFIs after influenza vaccination and to inform the risk communication messages to overcome vaccine hesitancy among pregnant women.

\section{Methods}

\section{Setting and study design}

The study was conducted at Rajavithi hospital in downtown Bangkok. Rajavithi is a tertiary level, 1200 bed hospital that began as a women's hospital but is now a full service medical facility. Approximately 150 women are seen for antenatal care (ANC) and 20 women deliver a baby on a daily basis. Among 5185 women with delivery outcomes at Rajavithi hospital in 2015, 80\% delivered full term, live infants, $19 \%$ delivered preterm infants, and $1 \%$ delivered stillborn infants.

We used a cohort study design to enroll women at the time of influenza vaccination (Influvac, Abbott Biologicals B.V., The Netherlands) [20] and follow them through the first four weeks post-vaccination to measure the frequency of AEFIs. We also abstracted delivery outcomes to measure the rate of adverse birth outcomes. There was no contemporaneous control group. However, the frequency of adverse birth outcomes among the study cohort was compared to the general population of women delivering at Rajavithi hospital during the previous year.

In preparation for a separate cohort study of the impact of influenza vaccination on birth outcomes, the hospital strengthened its influenza vaccination program by relocating the influenza vaccination site to the ANC clinic, providing educational seminars to hospital staff, including physicians, on the benefits and safety of the influenza vaccine, and increasing the communication materials posted around the ANC clinic.

\section{Enrollment criteria}

Pregnant women were approached for enrollment if they were $\geq 17$ weeks of pregnancy and $\geq 18$ years of age, prescribed vaccination with the Southern Hemisphere formulation of the inactivated, trivalent influenza vaccine (IIV3) on enrollment day, and were able to read and write in Thai. Pregnant women were excluded if they had received any vaccination in the past four weeks or were scheduled to receive any other vaccine on enrollment day or the next four weeks, had a history of Guillain-Barre syndrome or severe allergic reaction following any vaccination, had an apparent mental disability, or were not given a follow-up ANC appointment during which they would be able to return a study symptom diary.

\section{Definitions}

An AEFI was defined according to Thai national guidelines as any new symptom or abnormality occurring within four weeks after influenza vaccination [19]. A serious adverse event (SAE) was defined as any of the following: death, life-threatening illness; hospitalization or prolongation of hospitalization; permanent disability or permanent damage; congenital anomaly or birth defect; any illness requiring intervention to prevent permanent impairment or damage; or any other important medical event [21].

\section{Data collection}

Following written informed consent, study nurses administered a structured questionnaire to participants to collect demographic and health history data, including diagnoses of chronic diseases (asthma, chronic lung disease, chronic heart disease, kidney disease, liver disease, neurologic or neuromuscular disorders, hemoglobinopathy, metabolic disease, immunosuppressive conditions, HIV infection, or cancer). Study nurses instructed participants on how to report new symptoms or abnormalities using a daily diary, how to use a ruler to measure the diameter of redness and induration at the injection site, and how to take their body temperature using an axillary thermometer. Participants 
were asked to record the presence or absence of specific AEFIs known to be associated with vaccination to describe their perceived level of discomfort and inconvenience from each AEFI on a 3-point scale. The solicited AEFIs included soreness/pain and redness at injection site, induration around injection site, fever/feverish, headache, general weakness, muscle ache, nausea, and pruritus. Additionally, the participants were asked to measure body temperature once a day at the same time of day for the first four days after vaccination. Participants were also asked to record hospitalizations for any reason for four weeks following vaccination. Participants were also instructed to record any other new symptoms not specifically requested during the four weeks following vaccination. Participants were asked to return the diary at their subsequent ANC visit. Data from the diary were abstracted into a structured database for analysis.

Following enrollment and vaccination, participants were observed for $30 \mathrm{~min}$, as required by the national guidelines, at the ANC clinic for any AEFIs. On day 3 following vaccination, a study nurse phoned all participants to identify any AEFI requiring medical attention and to ensure that medical care was received. Within a few days of the expected delivery date, participants were phoned to determine whether the delivery had occurred, after which study nurses reviewed medical records to collect data on estimated gestational age and delivery outcomes. The delivery outcomes collected included miscarriage defined as premature loss of a fetus before 24 weeks of pregnancy, stillbirth defined as birth of a fetus from 24 weeks gestation showing no vital signs [22], prematurity defined as live birth at $<37$ weeks gestation [23], and term delivery defined as live birth at $\geq 37$ weeks), and birth weight. Any hospital visits or hospitalizations were verified using medical records.

\section{The diary}

A diary was used to collect information on the presence/ absence of the solicited AEFIs during the first four days, perceived discomfort, the number of days until the AEFI resolved, and AEFI management. Unsolicited AEFIs occurred during the first four days were recorded in a similar fashion as solicited AEFIs, except that they were recorded only when presence. An extra space was available to record any AEFI that occurred from day 5 up to 4 weeks. The following discomfort and inconvenience scale was used: mild (does not interfere with daily activity); moderate (affects daily activity); and severe (prevents daily activity) [24, 25]. AEFI management was self-reported, verified by chart review and categorized as requiring no management; over-the-counter medicine only; physician consultation with prescription for pain relief; physician consultation with prescription for pain relief plus other medicines; or hospitalization. The likely causal association between influenza vaccination and any SAE was graded by a study physician according to the WHO's criteria: certain or very likely, probably, possibly, unlikely, unrelated, or unclassifiable [21].

\section{Sample size}

A sample size of 289 was calculated to estimate the prevalence of an AEFI of $25 \%$, with a precision of \pm 5 percentage-points or an AEFI with a prevalence as low as $5 \%$ with a precision of \pm 3 percentage-points, assuming a Type I error rate of $5 \%$. To account for an expected loss to follow-up of 5\%, an enrollment goal of 305 pregnant women was estimated.

\section{Results}

Between July and November 2015, all 378 women with influenza vaccination prescription were screened for enrollment, of whom 308 (81\%) were eligible for inclusion. Of those who were eligible, 305 (99\%) consented to participate. All participants returned complete diaries, and 294 (96\%) had delivery outcome data collected. The median age of participants was 29 years (interquartile range [IQR] 24-34 years), and 271 (89\%) had completed secondary school or higher. Most $(184,60 \%)$ participants were multiparous, 223 (73\%) were in the third trimester (Table 1). Among those who were multiparous, 33 (18\%) reported at least one prior influenza vaccination before the current pregnancy. Pre-existing condition was reported by 20 (7\%) participants. Four participants had

Table 1 Characteristics of 305 pregnant women enrolled in the cohort

\begin{tabular}{ll}
\hline Characteristics at enrollment & $\begin{array}{l}\text { Frequency } \\
(\%)\end{array}$ \\
\hline $\begin{array}{l}\text { Age at enrollment (years) } \\
\leq 20\end{array}$ & $17(6)$ \\
$>20$ to 30 & $151(49)$ \\
$>30$ to 40 & $131(43)$ \\
$>40$ & $6(2)$ \\
Gestational age & \\
$\quad$ Second trimester & $83(27)$ \\
$\quad$ Third trimester & $222(73)$ \\
Education level & \\
Some or completed primary school & $34(11)$ \\
Completed secondary school & $74(24)$ \\
Completed diploma or high vocational school & $77(25)$ \\
Completed bachelor degree or higher & $120(39)$ \\
First pregnancy & $121(40)$ \\
Reported having underlying medical condition & $20(7)$ \\
Reported receiving influenza vaccination before this & $33(18)$ \\
pregnancy &
\end{tabular}

${ }^{\mathrm{a}}$ Among those who were multiparous 
allergy, 4 had metabolic disease, 3 had asthma, 2 had hemoglobinopathy, 2 had heart disease, 2 had HIV infection, and one each had liver disease, epilepsy, or depression.

Overall, 134 (44\%; 95\% confidence interval [CI] 38$50 \%$ ) participants reported $\geq 1$ AEFIs during the fourweek follow-up (Table 2). Of these, 120 (89\%) reported the AEFIs occurred on the day of or one day following vaccination. Almost all AEFIs (132, 99\%) occurred within four days following vaccination. The other two AEFIs occurred at 19 and 25 days following vaccination. The most common solicited AEFIs reported by participants were soreness at the injection site $(74,24 \%$; CI $19-29 \%)$, general weakness (50, 16\%; CI 12-21\%), muscle ache (49, 16\%; CI 12-21\%), and headache (45, $15 \%$; CI 11-19\%) (Table 2). Five (2\%; CI 0.5-4\%) participants reported having fever or feeling feverish, of whom, 4 (1\%; CI $0.3-3 \%)$ reported having a measured axillary temperature of $>37.5{ }^{\circ} \mathrm{C}$ starting on day 3 or day 4 while one reported feeling feverish and having the fever onset 25 days following vaccination. The four women with early fever onset had temperatures of $<39.0{ }^{\circ} \mathrm{C}$ and resolution of fever within $24 \mathrm{~h}$. Temperature was not measured for the participant with late fever onset and she did not seek care. Two participants reported a body rash as an unsolicited AEFI (0.7\%; CI $0-2 \%)$. Ten women (7\%) with AEFIs reported that the AEFIs affected their daily activities (Fig. 1a). All, except three AEFIs, spontaneously resolved. AEFIs were resolved within $24 \mathrm{~h}$ of onset in the majority of participants: $36(72 \%)$ of those with general weakness, 32 (71\%) of those with headache,
$12(71 \%)$ of those with nausea, $12(67 \%)$ of those with redness at injection site, 28 (57\%) of those with muscle ache, $4(57 \%)$ of those with induration at injection site, 41 (55\%) of those with sore at injection site, and $4(40 \%)$ of those with pruritus. In the two participants with body rash, resolution occurred 10 and 13 days after the onset. Two potential SAEs (decreased fetal movement and chorioamnionitis) were reported during the 4-week follow-up (0.7\%; CI 0-2\%) but determined to be unrelated to the vaccine by a study physician. No hospitalizations were identified (0\%; CI $0-1 \%)$.

There were 294 participants who were interviewed after delivery and had birth outcomes abstracted from the medical chart: 280 (95\%; CI 92-97\%) delivered full term, live infants; 12 (4\%; CI 2-7\%) delivered preterm live infants; $1(0.3 \%$; CI $0-2 \%)$ miscarried; and $1(0.3 \%$; CI $0-2 \%)$ had a stillbirth. The median birthweight of term and preterm infants were $3125 \mathrm{~g}$ (IQR 2928-3365) and $2521 \mathrm{~g}$ (IQR 2168-2851), respectively.

\section{Discussion}

We followed 305 Thai pregnant women prospectively for four weeks after influenza vaccination for solicited and unsolicited AEFIs and collected data on their delivery outcomes. Forty-four percent of women reported one or more AEFIs, but the majority of which were minor and self-limiting, and frequently reported after influenza vaccination in the general population. Fever occurred infrequently among participants, and none of the participants had axillary temperature $\geq 39$.

Table 2 Frequency of adverse events occurring within four weeks of influenza vaccination among 305 pregnant women, Bangkok, Thailand ${ }^{a, b}$

\begin{tabular}{|c|c|c|c|c|}
\hline \multirow[t]{2}{*}{ Symptoms } & \multicolumn{2}{|c|}{ Pregnant women, Bangkok } & \multirow{2}{*}{$\begin{array}{l}\text { Comparison group } 1^{d} \\
\%\end{array}$} & \multirow{2}{*}{$\begin{array}{l}\text { Comparison group } 2^{\mathrm{d}} \\
\%\end{array}$} \\
\hline & Frequency & $\%$ (95\% confidence interval & & \\
\hline One or more adverse events & 134 & $44(38.0,50.0)$ & NR & NR \\
\hline Pain/soreness at injection site & 74 & $24.3(19.4,29.1)$ & 36.4 & $1-10$ \\
\hline Redness at injection site & 18 & $5.9(3.2,8.6)$ & 1.9 & $1-10$ \\
\hline Induration/swelling at injection site & 7 & $2.3(0.6,4.0)$ & 2.1 & $1-10$ \\
\hline Body temperature $>37.5^{\circ} \mathrm{C}^{\mathrm{C}}$ & 4 & $1.3(0,2.6)$ & 1.6 & $1-10$ \\
\hline Headache & 45 & $14.8(10.8,18.8)$ & 15.9 & $1-10$ \\
\hline General weakness & 50 & $16.4(12.4,21.0)$ & 15.8 & $1-10$ \\
\hline Muscle ache & 49 & $16.1(11.9,20.2)$ & 16.4 & $1-10$ \\
\hline Nausea & 17 & $5.6(3.0,8.2)$ & $N R$ & NR \\
\hline Pruritus & 10 & $3.3(1.3,5.3)$ & NR & NR \\
\hline Rash & 2 & $0.7(0,2.3)$ & NR & NR \\
\hline
\end{tabular}

Abbreviations: NR not reported

${ }^{a}$ All except rash were solicited adverse events

${ }^{\mathrm{b}}$ In addition to the adverse events shown in the Table 2, two women had serious adverse events (decreased fetal movement and chorioamniitis) that were judged unrelated to vaccine

'Excluding one participant who reported a fever onset 25 days after vaccination whose body temperature was not measured

${ }^{\mathrm{d} C o m p a r i s o n}$ group data from Fluarix Quadrivalent package insert [26] (group 1) and Influvac Trivalent package insert [20] (group 2) 


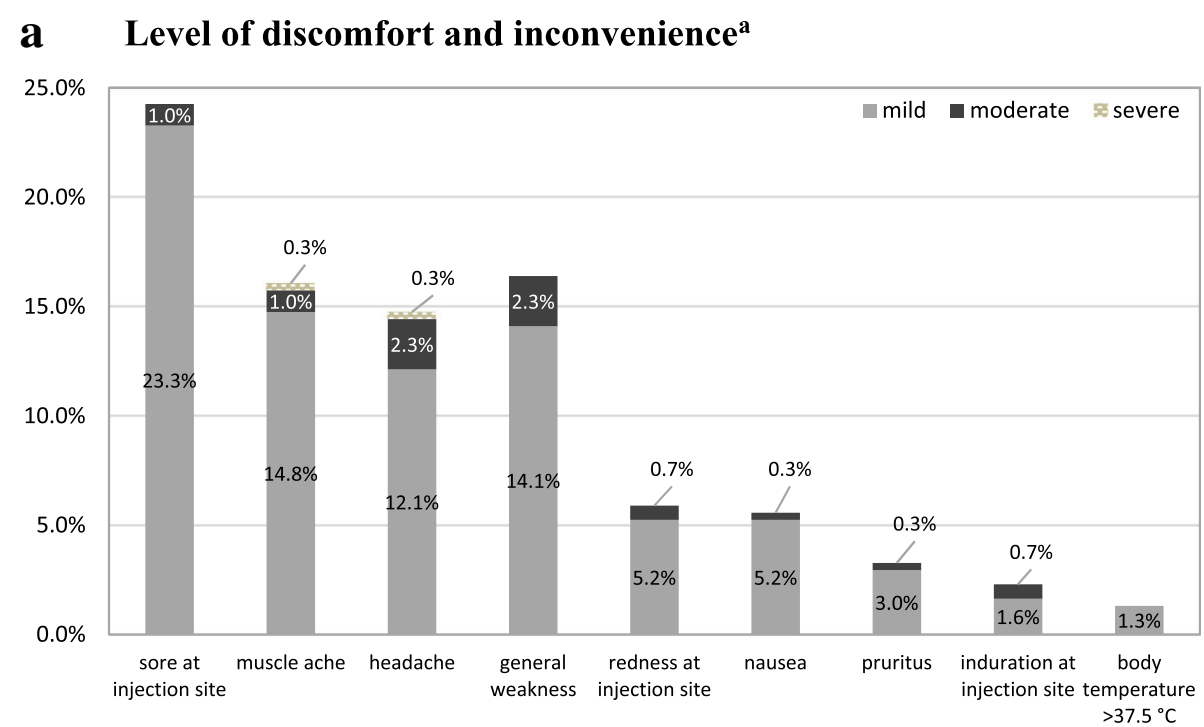

b Resolution ${ }^{b}$

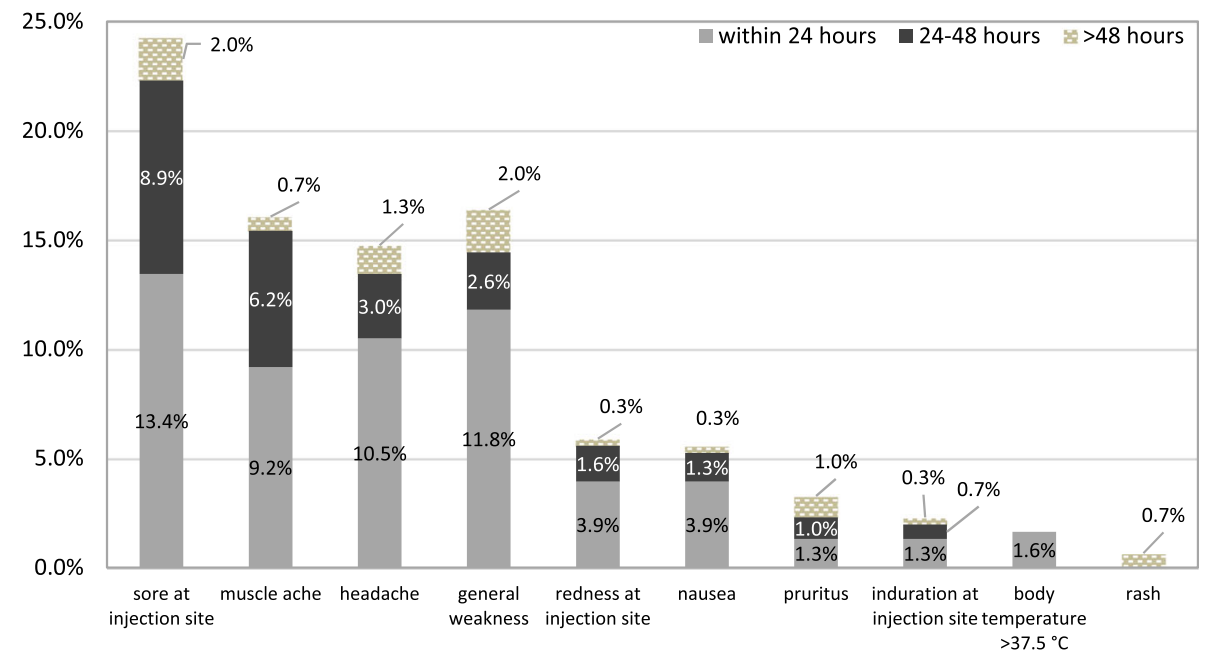

Fig. 1 Level of discomfort and inconvenience was self-reported and classified using a 3-point scale: a) mild, did not interfere with daily activity, b) moderate, affected daily activity, and c) severe, prevented daily activity. For redness and induration at/around injection site, the following classification was used: a) mild, reaction at injection of $<1 \mathrm{~cm}$ in diameter, b) moderate, reaction at injection between 1 and $<2 \mathrm{~cm}$ in diameter, and c) severe, reaction at injection that is $\geq 2 \mathrm{~cm}$ in diameter. For body temperature, the following classification was used: a) mild, temperature between 37.5 and $<39.0^{\circ} \mathrm{C}$, b) moderate, temperature between 39 and $<40{ }^{\circ} \mathrm{C}$, and c) severe, temperature $\geq 40{ }^{\circ} \mathrm{C}$. a In addition to the adverse events shown in the fig. 1b, there were one participant reported a fever onset 25 days after vaccination (resolved in 24 hours of onset), two participants who reported having rash (resolved 10 and 13 days following onset), and two participants with vaccine-unrelated serious adverse events (resolution could not be determined). b From symptom onset

$0{ }^{\circ} \mathrm{C}$. None of the women in our study experienced an SAE that was deemed vaccine-related.

The most common types and frequency of AEFIs identified prospectively in this cohort of Thai pregnant women were similar to those reported in clinical trials of inactivated influenza vaccine in non-pregnant adults. Compared with data from Fluarix Quadrivalent package insert, we found similar rates of induration/swelling, fever, headache, weakness, and muscle ache; a lower rate of soreness/pain; and a higher rate of redness (Table 2) [26]. We found similar rates of induration/swelling, fever, and redness, and a higher rates of soreness/pain, weakenss, muscle ache, and headache when compared our findings with data from Influvac Trivalent package insert [20]. Frequency of AEFIs in our study was higher than that reported in some other studies. In both a post-vaccination cluster survey of pregnant women in Laos and a study conducted among pregnant women in Australia, 13\% of pregnant women reported experiencing an AEFI [27, 28], although both of these studies followed women for AEFIs 
only up through seven days after vaccination compared to our month of follow-up, and neither used diary cards.

Although the study was not powered to estimate the frequency of rare events, the prevalence of stillbirth and miscarriage among the women in our study who received IIV3 was lower than that found among all pregnant women delivering at the hospital in the year prior to the study ( $80 \%$ delivered full term, live infants, $19 \%$ delivered preterm infants, and $1 \%$ delivered stillborn infants).

Several strengths of our study include: a defined cohort with a high rate of follow-up, a standardized diary to collect AEFI data, and characterization of AEFIs using standard definitions. In addition, chart abstraction was performed to collect selected delivery outcomes. However, several limitations should be considered when interpreting our findings. First, assessment of AEFIs was self-reported. Due to the wording on the symptom diary, the study could not differentiate between generalized myalgia and muscle ache at the site of injection and a misclassification between the two may have occurred. Second, the study was not powered to estimate the frequency of rare events and SAEs. Third, the study was designed to collect information only for up to four weeks following vaccination to avoid over burdening pregnant women keeping the diary. However, previous studies have shown that AEFIs from influenza vaccine usually begin soon after vaccination and last 1-2 days [24], so it is likely that the study captured most acute and common events. In addition, because impact of vaccination on delivery outcomes is an important issue in assessing vaccine tolerability and safety in pregnant women, we did collect data on delivery outcomes beyond the four-week follow-up window. Fourth, influenza vaccination history prior to this current pregnancy was self-reported and not verified. Participants may have mistakenly reported other vaccinations received as influenza vaccination; this might apply especially to multiparous participants who received the required tetanus toxoid vaccination during previous pregnancies. Lastly, there was no contemporaneous control group, but the frequency of most common AEFIs was compared to findings from similar studies $[19,20,26-28]$ and frequency of adverse delivery outcomes among the study cohort was compared to background frequency among the general population of women delivering at Rajavithi hospital during the previous year.

\section{Conclusions}

We found that influenza vaccine given in the second or third trimester of pregnancy was well tolerated by women in our study. Rates of reported AEFIs were consistent with those reported in prior studies and the large majority were mild and self-limiting. These findings support current recommendations by $\mathrm{WHO}$ and the Thailand MOPHfor influenza vaccination of pregnant women. However, these vaccine recommendations are only impactful if they are implemented and accepted by healthcare providers and pregnant women themselves. Communication messages underscoring the safety of influenza vaccination for pregnant women therefore should be tailored to healthcare providers, and pregnant women to increase vaccination uptake. Indeed this is already being undertaken by the Thailand National Immunization Program. In the future, AEFI systems for pregnant women in Thailand should be strengthened to permit prospective and continuous collection of vaccine safety data and monitoring for rarer events.

\section{Abbreviations}

AEFI: adverse event following immunization; ANC: antenatal care; Cl: 95\% confidence interval; IIV3: inactivated, trivalent influenza vaccine; IQR: interquartile range; MOPH: Ministry of Public Health; SAE: serious adverse event; WHO: World Health Organization

\section{Acknowledgements}

The authors thank all pregnant women who participated in this study. In addition, the authors would like acknowledge the help and support of the research team at Queen Sirikit National Institute of Child Health (Bangkok,

Thailand): Dr. Warunee Punpanich Vandepitte, Dr. Varaporn Sangtawesin, Dr. Meera Khorana, Dr. Wiboon Kanjanapattanakul, Somchit Ruenthong, Uraiwan Prachantasen, Naruemon Sassungnune, Jivanan Chompupuen, Wimolrat Thongngern, Patsada Somkhuntod, Saranya Winyawong, Sudarat Intranusorn, Apiradee Jamtiengtrong, Suchada Srisarang, Bajaree Chotpitayasunondh, Jiraporn Phourai, Rattanaporn Nartkul, Nongnuch Chanphet, Sarayut Sridontong, Chitdej Jirapong, Krongthong Jirapong, Anchalee Nontongpool, Hatairat Kunakronadul, Suthita Surinchai, Supang Prasongnak, Sasiprapa Dernchaiyaphum, Chamaiporn Khosasu, and Jaruwan Kamjohnjiraphun. Finally, we thank Amonlaya Chaiyakum, Duangporn Limsritrakul, and Waraporn Sakornjun of the Thailand Ministry of Public Health - U.S. Centers for Disease Control and Prevention Collaboration (Nonthaburi, Thailand) for their administrative and data management assistance.

\section{Funding}

This project was funded by U.S. Centers for Disease Control and Prevention through cooperative agreement 5U01GH000152.

\section{Availability of data and materials}

The datasets used and/or analysed during the current study are available from the corresponding author on reasonable request.

\section{Disclaimer}

The findings and conclusions in this report are those of the authors and do not necessarily represent the official position of the U.S. Centers for Disease Control and Prevention or the U.S. Government.

\section{Authors' contributions}

SA contributed to participant enrollment, data collection, drafted the initial manuscript, reviewed and approved the final manuscript as submitted. WK conceptualized and designed the study, designed data collection instruments, coordinated and supervised data collection, carried out all data analyses, reviewed and revised the manuscript, and approved the final manuscript as submitted. PS conceptualized and designed the study, assisted with the design of data collection instruments, coordinated and supervised data collection, reviewed and approved the final manuscript as submitted. DD assisted with the design of data collection forms, coordinated and supervised data collection, reviewed and approved the final manuscript as submitted. SK, PP, and NS contributed to participant enrollment, data collection, reviewed and approved the final manuscript as submitted. TC conceptualized and designed the study, reviewed and approved the final manuscript as submitted. FS assisted with the design of data collection 
instruments, provided inputs in the data analysis process, reviewed the manuscript, and approved the final manuscript as submitted. KL conceptualized and designed the study, assisted with the design of data collection instruments, provided intputs in the data analysis process, reviewed and revised the manuscript, and approved the final manuscript as submitted. All authors have given final approval of the version to be published, have participated sufficiently in the work to take public responsibility for appropriate portions of the content, and agreed to be accountable for all aspects of the work in ensuring that questions related to the accuracy or integrity of any part of the work are appropriately investigated and resolved.

\section{Ethics approval and consent to participate}

This study was approved by the ethics committees of the Thailand MOPH (Nonthaburi, Thailand) and Rajavithi hospital (Bangkok, Thailand) with the U.S. Centers for Disease Control and Prevention (Atlanta, GA, USA) reliance on the Thai MOPH ethics committee. All participants in this study provided written, informed consent to participate.

\section{Consent for publication}

Not applicable.

\section{Competing interests}

The authors declare that they have no competing interests.

\section{Publisher's Note}

Springer Nature remains neutral with regard to jurisdictional claims in published maps and institutional affiliations.

\section{Author details}

${ }^{1}$ Rajavithi Hospital, Bangkok, Thailand. ${ }^{2}$ Influenza Program, Thailand Ministry of Public Health - U.S. Centers for Disease Control and Prevention Collaboration, Nonthaburi, Thailand. ${ }^{3}$ Queen Sirikit National Institute of Child Health, Bangkok, Thailand. ${ }^{4}$ Influenza Division, U.S. Centers for Disease Control and Prevention, Atlanta, USA.

\section{Received: 8 January 2018 Accepted: 16 March 2018}

Published online: 23 April 2018

\section{References}

1. WHO recommends seasonal influenza vaccination to pregnant women as the highest priority [http://www.who.int/immunization/newsroom/ newsstory_seasonal_influenza_vaccination_pregnancy/en/]. Accessed 23 Mar 2018

2. Seasonal influenza [http://beid.ddc.moph.go.th/beid_2014/th/diseases/253]. Accessed 23 Mar 2018

3. Neuzil KM, Reed GW, Mitchel EF, Simonsen L, Griffin MR. Impact of influenza on acute cardiopulmonary hospitalizations in pregnant women. Am J Epidemiol. 1998;148(11):1094-102.

4. Dodds L, McNeil SA, Fell DB, Allen VM, Coombs A, Scott J, MacDonald N. Impact of influenza exposure on rates of hospital admissions and physician visits because of respiratory illness among pregnant women. CMAJ. 2007;176(4):463-8.

5. Fell DB, Azziz-Baumgartner E, Baker MG, Batra M, Beauté J, Beutels P, Bhat N, Bhutta ZA, Cohen C, De Mucio B, et al. Influenza epidemiology and immunization during pregnancy: final report of a World Health Organization working group. Vaccine. 2017;35(43):5738-50.

6. Prapasiri P, Greenbaum A, Yoocharoen P, Ditsungneon D, Dawood FS, Muangchana C, Olsen SJ. Knowledge, attitudes, and practices about influenza vaccination among pregnant women in Thailand. Vaccine. 2016; 34(18):2141-6.

7. Jamieson DJ, Kissin DM, Bridges CB, Rasmussen SA. Benefits of influenza vaccination during pregnancy for pregnant women. Am J Obstet Gynecol. 2012;207(3 Suppl):S17-20.

8. Keller-Stanislawski B, Englund JA, Kang G, Mangtani P, Neuzil K, Nohynek H, Pless R, Lambach P, Zuber P. Safety of immunization during pregnancy: a review of the evidence of selected inactivated and live attenuated vaccines. Vaccine. 2014;32(52):7057-67.

9. Moro PL, Broder K, Zheteyeva Y, Walton K, Patricia Rohan P, Sutherland A, Guh A, Haber P, DeStefano F, Vellozzi C. Adverse events in pregnant women following administration of trivalent inactivated influenza vaccine and live attenuated influenza vaccine in the vaccine adverse event reporting system, 1990-2009. Am J Obstet Gynecol. 2011;204(146):e1-7.

10. Munoz FM, Greisinger AJ, Wehmanen OA, Mouzoon ME, Hoyle JC, Smith FA, Glezen WP. Safety of influenza vaccination during pregnancy. Am J Obstet Gynecol. 2005;192(4):1098-106.

11. Zaman K, Roy E, Arifeen SE, Rahman M, Raqib R, Wilson E, Omer SB, Shahid NS, Breiman RF, Steinhoff MC. Effectiveness of maternal influenza immunization in mothers and infants. Engl J Med. 2008;359(15):1555-64.

12. Tapia MD, Sow SO, Tamboura B, Tégueté I, Pasetti MF, Kodio M, Onwuchekwa U, Tennant SM, Blackwelder WC, Coulibaly F, et al. Maternal immunisation with trivalent inactivated infl uenza vaccine for prevention of infl uenza in infants in Mali: a prospective, active-controlled, observer-blind, randomized phase 4 trial. Lancet Infect Dis. 2016;16(9):1026-35.

13. Steinhoff MC, Katz J, Englund JA, Khatry SK, Shrestha L, Kuypers J, Stewart L, Mullany LC, Chu HY, LeClerg SC, et al. Year-round influenza immunisation during pregnancy in Nepal: a phase 4, randomised, placebo-controlled trial. Lancet Infect Dis. 2017;17(9):981-9.

14. Mahdi SA, Treurnicht F, Ortiz JR, Venter M, Violari A, Neuzil KM, Simões EA, Klugman KP, Nunes MC, Maternal Flu Trial (Matflu) Team. Influenza vaccination of pregnant women and protection of their infants. N Engl J Med. 2014;371(10):918-31.

15. Thompson MG, Li DK, Shifflett $P$, Sokolow LZ, Ferber JR, Kurosky S, Bozeman S, Reynolds SB, Odouli R, Henninger ML, et al. Effectiveness of seasonal trivalent influenza vaccine for preventing influenza virus illness among pregnant women: a population-based case-control study during the 2010-2011 and 2011-2012 influenza seasons. Clin Infect Dis. 2014:58(4):449-57.

16. Owusu JT, Prapasiri P, Ditsungnoen D, Leetongin G, Yoocharoen P, Rattanayot J, Olsen SJ, Muangchana C. Seasonal influenza vaccine coverage among high-risk populations in Thailand, 2010-2012. Vaccine. 2015;33(5):742-7.

17. Naleway AL, Irving SA, Henninger ML, Li DK, Shifflett P, Ball S, Williams JL, Cragan J, Gee J, Thompson MG, et al. Safety of influenza vaccination during pregnancy: a review of subsequent maternal obstetric events and findings from two recent cohort studies. Vaccine. 2014;32(26):3122-7.

18. Nordin JD, Kharbanda EO, Vazquez Benitez G, Lipkind H, Vellozzi C, Destefano F. Vaccine safety datalink: maternal influenza vaccine and risks for preterm or small for gestational age birth. J Pediatr. 2014;164(5):1051-7.

19. Bureau of Epidemiology, Department of Disease Control. Surveillance and investigation of adverse events following immunization (AEFI). 2nd ed. Nonthaburi: The War Veterans Organization of Thailand Printing; 2008.

20. Influvac(R) 2017 [package insert]. https://www.scribd.com/document/ 347239541/Influvac-Insert-2017. Accessed 23 Mar 2018.

21. Collet JP, MacDonald N, Cashman N, Pless R. Advisory committee on causality assessment: monitoring signals for vaccine safety: the assessment of individual adverse event reports by an expert advisory committee. Bull World Health Organ. 2000;78(2):178-85.

22. Nguyen RHN, Wilcox AJ. Terms in reproductive and perinatal epidemiology: I. Reproductive terms. J Epidemiol Community Health. 2005:59:916-9.

23. WHO Regional Strategy on Sexual and Reproductive Health. Definitions and indicators in family planning, maternal and child health and reproductive health. Denmark: World Health Organization Regional Office for Europe; 2001. p. 1-41.

24. Giezeman KM, Nauta J, de Bruijn IA, Palache AM. Trivalent inactivated subunit influenza vaccine Influvac ${ }^{\oplus}$ : 30-year experience of safety and immunogenicity. Vaccine. 2009;27(18):2414-7.

25. Adverse Event Following Immunization (AEFI) Case Report Form. http:// www.bccdc.ca/resourceresource-gallery/Documents/ Guidelines\%20and\%20Forms/Forms/Immunization/Vaccine\%20Info/ AEFICaseReportForm.pdf. Accessed 23 March 2018.

26. Fluarix(R) [package insert] [https://www.fda.gov/downloads/ BiologicsBloodVaccines/Vaccines/ApprovedProducts/UCM335392.pdf].

27. Regan AK, Tracey L, Blyth CC, Mak DB, Richmond PC, Shellam G, Talbot C, Effler PV. A prospective cohort study comparing the reactogenicity of trivalent influenza vaccine in pregnant and non-pregnant women. BMC Pregnancy Childbirth. 2015;15:61.

28. Phengxay M, Mirza SA, Reyburn R, Xeuatvongsa A, Winter C, Lewis H, Olsen SJ, Tsuyuoka R, Khanthamaly V, Palomeque FS, et al. Introducing seasonal influenza vaccine in low-income countries: an adverse events following immunization survey in the Lao People's Democratic Republic. Influenza Other Respir Viruses. 2015;9(2):94-8. 